\title{
La psicología en la ciudad de Los Ángeles, Chile. Una historia local desde las trayectorias biográficas de los/as psicólogos/as Psychology in Los Ángeles, Chile. A Local History From the Biographical Trajectories of Psychologists
}

\author{
Nicol E. Meza ${ }^{a}$, Diego I. Quezada ${ }^{\mathrm{a}}, \&$ Rodolfo E. Mardones ${ }^{\mathrm{b}}$ \\ ${ }^{a}$ Universidad Santo Tomás, Los Ángeles, Chile. \\ ${ }^{b}$ Universidad Austral de Chile, Valdivia, Chile.
}

Las investigaciones realizadas sobre historia de la psicología en Chile se han caracterizado por ser historias generales e inscritas en una pretensión de universalidad. A partir de esto y con el propósito de movilizar la visibilidad de la historia de la disciplina a espacios locales, la presente investigación tiene por objetivo describir las trayectorias biográficas de psicólogos/as, aportando elementos desde una perspectiva descriptiva de carácter cualitativa con orientación biográfica. Se expone un estudio de caso en una ciudad del sur de Chile, utilizando fuentes orales recolectadas a través de entrevistas en profundidad. Los principales resultados se exponen en torno a las categorías de formación, adscripción teórica y práctica profesional de los/as entrevistados/as. Se concluye que las trayectorias biográficas de los/as psicólogos/as articulan prácticas y significados de la institucionalización de la disciplina en la ciudad.

Palabras clave: psicología, historia local, trayectorias biográficas.

The investigations realized on history of the psychology in Chile have been characterized for being general histories inscribed in a logic with pretensions of universality. From this point, and with the intention of mobilizing the visibility of the history of the discipline to local spaces, the present investigation aims to describe the biographical paths of psychologists, contributing elements from a descriptive qualitative perspective of character with biographical orientation. A study of case is exposed in a city of the south of Chile, using oral sources collected through in-depth interviews. The main results are exposed around the categories of training, theoretical ascription and professional practice of the interviewees. It is concluded that the biographical trajectories of psychologists articulate practices of the institutionalization of the discipline in the city.

Keywords: psychology, local history, biographical trajectories.

Contacto: R. E. Mardones. Escuela de psicología Universidad Austral de Chile, isla Teja s/n, Valdivia. Correo electrónico: rodolfo.mardones@uach.cl

Cómo citar: Meza, N. E., Quezada, D. I., \& Mardones, R. E. (2018). La psicología en la ciudad de Los Ángeles (Chile). Una historia local desde las trayectorias biográficas de los/as psicólogos/as. Revista de Psicología, 27(2), 1-12.

http://dx.doi.org/10.5354/0719-0581.2019.52313 


\section{Introducción}

Los estudios sobre la historia de la psicología han recibido una atención importante en los últimos años. Los tópicos de interés han sido el estudio de los pioneros/as, los laboratorios experimentales, los primeros programas de formación de los psicólogos, la producción científica, historia del colegio de psicólogos, la recepción de teorías y autores, entre otros (e.g., Bravo, 2004; Bravo-Valdivieso \& Tschorne Tetelman, 1969; Laborda \& Quezada, 2010; Luco, 2010; Poblete, 1980; Ruperthuz Honorato, 2015; Salas, 2013; Salas \& Lizama, 2013; Toro \& Villegas, 2001; Villegas, Marassi, \& Toro, 2003a, 2003b; Winkler, 2007). Estos estudios han contribuido en el establecimiento de periodizaciones y recopilaciones de datos que resultan fundamentales para sustentar investigaciones posteriores. Estas publicaciones, en general, dan cuenta de eventos fundantes de la disciplina en Santiago de Chile, a propósito de que la formación de psicólogos/as tuvo su origen en la capital del país. En estas construcciones de la historia de la disciplina, los acontecimientos se ordenan en una lógica similar a la advertida por Danziger (1979): en un progreso de acumulación lineal, con base en la descripción objetiva de fuentes y sustentada en la verificación documental. Sin embargo, existen ciertas particularidades que se escapan al foco de estos estudios y que se presentan como espacios anónimos y no articulados para las construcciones históricas de la disciplina.

Aunque de forma menos central, la psicología ha tenido aproximaciones que focalizan en la dimensión local como aspecto articulador de estudios que se orientan a historizar la disciplina. Este enfoque aseguraría una nueva comprensión de la historia que se caracteriza por establecer límites que permitan una reducción de escala, pero que, al mismo tiempo, implique la consciencia de su artificialidad (Salas, 2016).

En términos empíricos, Klappenbach (2011, 2016), identifica trabajos iniciales en esta comprensión de la historia, remitiendo a un trabajo inicial, desarrollado en Alemania por Sprung, Sprung y Schönpflug. Destaca la historia regional como una perspectiva flexible y transversal a las distintas perspectivas de la historia, ya que presenta utilidad para investigar la psicología en contextos locales. Se advierte que los estudios asociados a esta perspectiva de la historia reportan un interés inicial por un espacio geográfico específico o por un determinado campo o subcampo de la psicología. En este sentido, la delimitación de lo local puede estar determinado por el tamaño de los trabajos, las fuentes, la diferenciación sobre quien realiza la historia, las características desde donde se escribe, la consideración de modelos previos de desarrollo de la historia de la psicología, su potencial como alternativa reflexiva para el estudio del paisaje cultural, y el rescate de omisiones o silenciamientos que ocurren en la historia de la psicología (Salas, 2016). El impacto de esta concepción de la historia sugiere la detención en lo local, cuestión que puede tomar distintos enfoques para delimitar su aproximación empírica (Mardones Barrera, 2016a). Lo anterior exige acudir a desafíos metodológicos (Klappenbach, 2016), que no solo deben estar basados en un propósito verificacionista, sino que deben orientarse a conocer en profundidad el fenómeno estudiado, muchas veces en desmedro de su extensión y generalización (Mardones Barrera, 2016b).

De acuerdo con la literatura, es posible reconocer estudios que se detienen en historizar la psicología a partir de la reducción de escala geográfica, la institucionalidad, los objetos-cosas y las figuras emblemáticas o pioneros. Estos tópicos han sido centrales para trazar los límites de lo local y, en la mayoría de los casos se presentan superpuestas.

La reducción de la escala geográfica como criterio para circunscribir historias acotadas a mapas geopolíticos puede graficarse con estudios emplazados en países y ciudades periféricos. Esta perspectiva ha recibido una atención amplia $\mathrm{y}$, a modo de ejemplo, se puede mencionar el desarrollo de la disciplina en distintos países respecto a la historia mundial de la psicología, por ejemplo en Argentina (Rossi, Ibarra, \& Ferro, 2009; Saforcada, 2008), en Paraguay (García, 2007), en Perú (Alarcón, 1980a; Benites Morales \& Zapata Ponce, 2009), en República Dominicana (Rodríguez Arias, 2009), en Cuba (De la Torre Molina, 2009), en Bolivia (Aguilar, 1983; Vía Orellana, 2000), en Brasil (Rodrigues Soares, 2010), y en Colombia (Ardila, 1973), entre otros. Con una mayor reducción de la escala geográfica se puede mencionar el desarrollo de la psicología en la ciudad de Encarnación, Paraguay (García, 2012), en Cuenca, Ecuador (Salinas Villacrés \& Prado Cabrera, 2017), en Arequipa (Arias Gallegos, 2016), en Córdoba (Scherman, 2014), y en la Plata (Dagfal, 2014).

La institucionalidad también se ha considerado 
como criterio para circunscribir historias acotadas a espacios organizacionales, académicos y profesionales, enmarcando estudios que se refieren a la historia de una carrera de psicología, una universidad, la producción científica, colegios de psicólogos u otras iniciativas gremiales. Por ejemplo, se puede mencionar el caso de la carrera de psicología en la Universidad de Chile (Ligüeño Espinoza \& Parra Moreno, 2007), en las universidades privadas de Bolivia (Schulmeyer, 2013), en universidades de Argentina (Gallegos, 2005; Klappenbach, 2011; Ventura, 2004) y Brasil (Polanco \& Lopes Miranda, 2014), en una universidad peruana (Barboza-Palomino, 2016) y en universidades paraguayas (García, 2011). En cátedras específicas podemos mencionar el segundo curso de psicología en la Universidad de Buenos Aires (Klappenbach, 1994), la de psicología experimental en la Universidad Católica de Asunción (García, 2010), y en sociedades científicas como la Sociedad Interamericana de Psicología (Gallegos, 2012; Maluf, 2012).

En Chile, los estudios sobre este tópico se han detenido en eventos de la Universidad de Chile: el curso de formación de psicólogos en el Instituto Pedagógico (1947), la creación del Instituto de Psicología, y la formación de psicólogos en la primera carrera de psicología (Ligüeño Espinoza \& Parra Moreno, 2007; Salas \& Lizama, 2013). La Pontificia Universidad Católica también destaca como otra institución importante para los inicios de la psicología en el país con la creación de su escuela de Psicología en 1956 (Salas \& Lizama, 2013).

Otros aspectos indagados desde una perspectiva institucional como delimitación de lo local refiere a hechos históricos específicos. En el caso chileno se refiere a eventos de violencia política en la dictadura militar (1973-1990), estos son: el cierre de la carrera de psicología y exilio de profesores en la Universidad de Chile (Ligüeño Espinoza \& Parra Moreno, 2007), las operaciones psicológicas militares, en contra de la población, a cargo de Hernán Tuane (Salas \& Lizama, 2013; Vetö Honorato, 2013), y el rol del Colegio de Psicólogos frente a la vulneración de los derechos humanos (Luco, 2016).

Un último tópico referido a lo institucional es la implementación del modelo económico neoliberal en la dictadura militar chilena, la que posibilitó modificaciones estructurales a la institucionalidad nacional. En lo que respecta al sistema de educación universitaria, en 1981 comenzó el proceso de la privatización de la educación y creación de universidades privadas, las cuales elevaron de gran manera el número de estudiantes matriculados (Villegas, 2016).

Por último, cabe destacar los trabajos, que, para entender lo local, recurren a figuras emblemáticas y pioneras de la psicología. En este contexto, se reconoce el rol de pioneros/as en la disciplina a sujetos que realizaron algún aporte a la psicología: autores de libros, artículos u otros escritos susceptibles de encuadrarse en el área de la psicología; alguna contribución de manera independiente; difusores de una idea o de las teorías en el medio local; el ejercicio de una investigación activa; la colaboración activa en los distintos niveles de organización institucional que hicieron posible el afianzamiento de la psicología; fundadores de los primeros laboratorios psicológicos $\mathrm{y} / \mathrm{o}$ institutos de enseñanza (García, 2007).

Se ha destacado la figura de personajes y contribuyentes a los distintos campos de la disciplina, como, por ejemplo: Martín-Baró en El Salvador (Barrero C., 2012), Mira y López, en España y Brasil (Hoffmann, Tortosa, \& Carbonell Vayá, 1994), Helena Antipof en Brasil (Campos, 2003), Walter Blumenfeld en Perú (Alarcón, 1980b), Mercedes Rodrigo (Ardila, 1988), entre muchos otros de una larga lista. Por otro lado, intentando hacer justicia en la selecta lista de hombres pioneros, se ha destacado el rol de mujeres pioneras de la psicología, entre ellas Lola Hoffmann, Héliettè Saint Jean y Vera Kardonsky (Winkler \& Reyes-Espejo, 2015).

En Chile, se ha destacado la figura de Rómulo Peña por su responsabilidad en la instalación del primer laboratorio de psicología experimental en la Escuela Normal de Copiapó (Salas Contreras, 2012), Jorge Enrique Schneider, Wilhelm Mann y Valentín Letelier (Salas Contreras, 2012), Amanda Labarca (Salas, Mardones, Gallegos, \& Ponce, 2014), Andrés Bello (Salas y Lizama, 2013), y Juan Serapio Lois (Salas, 2013). También se ha resaltado al médico chileno Germán Greve Schlegel y su temprano vínculo con el psicoanálisis (Ruperthuz Honorato, 2014), y a Fernando Allende Navarro, médico y psicoanalista formado en Europa que regresa a Chile en 1925 (León, 1982; Ruperthuz Honorato, 2014). Finalmente se destaca a Sergio Yulis como una figura importante para instalación del conductismo en Chile (Quezada, Vergés, \& Laborda, 2014).

De acuerdo con lo anterior, son muchos y variados los esfuerzos por recoger una perspectiva local 
de la psicología, en el amplio sentido del uso del término. En este caso, la nominación de local reconoce el origen situado de cada versión de la historia de la disciplina (Mardones Barrera, 2016b). Se toma la precaución que Danziger (1979) sostiene sobre la tendencia de tratar la evidencia histórica como si fuera un caudal de hechos objetivos, desconociendo que se trata de reconstrucciones mediadas por intereses de los implicados. El autor sostiene que la relación entre generaciones sucesivas es reducida a la de pioneros y continuadores, y, a partir de estas figuras, se historiza la psicología. Por ejemplo, a partir del supuesto acto de creación de Wundt, como un proceso de desarrollo interno dentro de la disciplina, sin entender el rol crítico de los factores externos a esta. Este punto implica una comprensión de la historia de la psicología que considere estos factores externos en relación con los internos. En este sentido, se entiende que toda configuración histórica de la psicología es una historia local debido a su origen sociocultural y territorial especifico (Mardones Barrera, 2016b). La preocupación por lo local, tanto a nivel interno como externo a la psicología, podría tematizarse como historias locales, microhistorias o historias alternativas (Sanmartín Ascaso, 1995), denominaciones que comparten la preocupación de resaltar historias no narradas e invisibilizadas por una narrativa general. En cambio, se abandonan los eventos regulares para detenerse en elementos subjetivos y cotidianos (Man, 2013).

La historia local es una perspectiva desarrollada para dar un impulso sustantivo al reconocimiento de realidades subalternas en contraste con generalizaciones y narrativas heroicas, y busca atender los procesos históricos como una historia cultural que rescate los problemas desde la perspectiva de los involucrados (Aguirre Rojas, 2003). Ginzburg (1999) señala la importancia de retomar elementos documentales, biográficos y orales que permitan desarrollar esta perspectiva de la historia. El autor expone que en algunos estudios biográficos se ha demostrado que, "en un individuo carente en sí de relieve y por ello representativo, pueden investigarse las características de todo un estrato social en un determinado período histórico" (Ginzburg, 1999, p. 9). En este sentido, la investigación histórica cambia, dejando atrás la memoria colectiva de los grandes eventos y los grandes héroes. En cambio, valora los procesos sociales, los hechos cotidianos y las vicisitudes anónimas (Pasek de Pinto,
2006), sin perder de vista las posibles relaciones y/o conexiones que, limitadamente, los investigadores pueden pesquisar. En relación con lo anterior, surgen las siguientes preguntas de investigación: $¿$ es posible reconstruir historias locales desde la perspectiva de sus actores?, ¿cuáles son las trayectorias biográficas de psicólogos/as en un contexto local y periférico?

\section{Método}

Para responder nuestras preguntas de investigación, el método utilizado en esta investigación es de tipo cualitativo con enfoque descriptivo (Mardones, Ulloa Martínez, \& Salas, 2018). Se utilizó el método biográfico, operativizado mediante relatos de vidas (Pujadas, 2000; Sanz Hernández, 2005; Valles, 2007). Se recogió la narración biográfica de cinco participantes para la obtención de información acorde a la pregunta de investigación (Pujada, 2000). La recolección de datos, para la construcción de relatos de vida, se realizó por medio de entrevistas en profundidad, dirigidas hacia la comprensión de las perspectivas que tienen los informantes respecto a sus vidas, experiencias o situaciones, tal como la expresan sus propias palabras (Mallimaci \& Giménez Béliveau, 2006). En las entrevistas se solicitó a los/as participantes relatar su trayectoria personal desde el ingreso a la carrera de psicología hasta la actualidad. Todas las entrevistas se llevaron a cabo durante el año 2015 y se desarrollaron como una conversación abierta, orientada por una pauta temática compuesta de los siguientes tópicos: ingreso a la universidad, formación académica de pregrado y posgrado, inserción profesional y ejercicio de la profesión.

\section{Caso de estudio y participantes}

La investigación se desarrolló en la ciudad de Los Ángeles en la región del Biobío. Esta región del país es importante en el contexto nacional, por su rol en la formación de la república y por su condensación de relaciones políticas y económicas. También tiene un papel relevante en la formación de psicólogos/as, alojando la cuarta carrera de psicología del país en la Universidad de Concepción, a partir de 1985; después de la Universidad de Chile (1947), la Universidad Católica (1954) y la Universidad de la Frontera en Temuco (1982) (Salas \& Lizama, 2013).

Los Ángeles es una ciudad periférica de la 
capital regional del Biobío (Concepción) en el centro-sur de Chile. Su relación con la psicología se ha ido forjando a propósito de la incorporación de egresados de las distintas universidades mencionadas anteriormente. Es a propósito de esta posición periférica que se selecciona como caso de estudio relevante para la perspectiva de la historia local de la psicología.

La selección de los participantes se llevó a cabo mediante un muestreo por conveniencia basado en criterios de inclusión (Pérez-Luco Arenas, Lagos Gutiérrez, Mardones Barrera, \& Sáez Ardura, 2017), estos fueron: ser psicólogo/a titulado/a, la fecha de titulación hasta la década de 1990, y que ejercieron o estuvieran ejerciendo la profesión en la ciudad de Los Ángeles. El contacto con cada participante se concretó mediante el uso de técnica de bola de nieve (Pérez-Luco Arenas et al., 2017), a través de la mediación de un informante clave que sugirió a otros participantes. Se entrevistó un total de cinco psicólogos/as y como consideraciones éticas del estudio, se explicitó el propósito de la investigación a los participantes, se clarificó el uso de la información, se aseguró la voluntariedad en la participación y la confidencialidad de datos personales, lo cual se concretó por medio de la firma de un consentimiento informado individual.

\section{Análisis de datos}

Se realizó un análisis de contenido en tres fases: descubrimiento, codificación y relativización de los datos (Taylor \& Bogdan, 1994). En primera instancia se realizó una lectura reiterada de los datos para poder reducir y ordenar la información. De este modo se encontraron los temas relevantes a considerar. En la fase de codificación se procedió a agrupar por códigos y categorizar los datos recolectados. En la fase final analizaron los datos anteriormente codificados para encontrar las relaciones entre relatos y poder responder a la pregunta de investigación.

\section{Resultados}

Las trayectorias biográficas de los psicólogos fueron organizadas en tres categorías: formación académica y profesional, adscripción teórica y prácticas profesionales. La primera se refiere al proceso en que los psicólogos se formaron durante el pregrado; la segunda se refiere a adhesión profesional a alguna perspectiva teórica para dar explicación a los procesos psicológicos. Por último, la práctica profesional consiste en la trayectoria laboral de los entrevistados luego del egreso de su formación de pregrado.

\section{Formación académica y profesional}

El ingreso a la educación superior de los entrevistados fue entre los años 1971 y 1989, período que coincide con el gobierno de la Unidad Popular, el golpe de Estado, la dictadura militar y la instalación del modelo neoliberal en el país. Las casas de estudio desde las que egresaron los participantes fueron Universidad de Chile (Santiago), Universidad Católica (Santiago), Universidad de Concepción (Concepción) y Universidad Central (Santiago).

Según los participantes, el ingreso a la carrera de psicología estuvo estimulado por la influencia de los ramos de psicología de una carrera universitaria diferente o el interés por las mallas curriculares, y estuvo condicionado por la prueba de aptitud académica. Esto refleja las dificultades que, para los interesados/as, comenzaba a evidenciar el sistema de selección universitaria, sumado al esfuerzo de traslado que debían hacer a las ciudades centrales donde se impartía la carrera de psicología.

Por otro lado, al encontrarse en el contexto de dictadura, los participantes se vieron envueltos en diferentes problemáticas y un ambiente de represión política que impactó de manera directa en el ámbito educativo:

... cerraron las escuelas y yo tenía que dar mi examen de grado ... entonces, claro, fue absolutamente marcado por el golpe porque la escuela desapareció, alcancé a dar mi examen de grado el 17 de julio del 74 (participante 4).

... el primer mes de clases me quedó la escoba, yo vi la realidad así ...: protestas, gente corriendo, el año anterior habían matado a un alumno de ingeniería (participante 5).

La experiencia de la dictadura militar es planteada de forma central en el período de formación profesional, marcando tempranamente la trayectoria de los participantes al insertarse en el mundo universitario, lo cual, en algunos casos, significó un contraste abrupto respecto de la experiencia familiar y educativa previa a la universitaria.

... yo vivía en una burbuja en la ciudad ... 
totalmente alejada de la realidad, tenía poco acceso a la "realidad" producto de mi entorno familiar, se hablaba un poco, pero ustedes entenderán que en el colegio había pocos compañeros que hablaban del tema de la dictadura de Pinochet (participante 5).

Otro asunto destacado, en relación con la formación, es la organización curricular de las carreras. $\mathrm{Al}$ respecto, los/as entrevistados/as plantean que las asignaturas eran similares en los distintos planteles educativos. Sin embargo, refieren algunas diferencias en la formación curricular específica, donde en alguno de los casos, como en la Universidad Central, además de tener área clínica, organizacional y educacional, existían contenidos sobre psicología comunitaria en el período estudiado.

También se destaca, para este incipiente período de formación en psicología, el protagonismo de los estudiantes respecto de su formación. Un/a entrevistado/a reporta que, en la Universidad de Concepción, los estudiantes abrieron instancias de encuentro y diálogo, a pesar de las restricciones del estado de excepción:

... nosotros, en Concepción, creamos, organizamos, el primer encuentro nacional de estudiantes de psicología, lo hicimos nosotros, y de ahí para adelante, se instauró la tradición de todos los años hacer un encuentro nacional de estudiantes de psicología (participante 5).

De acuerdo con los/as entrevistados/as, la participación estudiantil aumentó en los últimos años de la dictadura, así también, la diversificación temática de la formación en psicología se vería acentuada debido al retorno de profesores exiliados y la reconquista de la libertad de expresión, lo cual impactó directamente en la formación de las nuevas generaciones.

\section{Adscripción teórica}

De acuerdo con la experiencia de los/as entrevistados/as, las carreras de psicología entregaban una formación curricular en las principales corrientes vigentes en la época: fenomenología, psicoanálisis, conductismo e, incipientemente, humanismo. Considerando esta formación y la inmersión profesional de los participantes, reportan que hubo ciertas orientaciones y personas que influyeron en su decisión sobre las perspectivas teóricas desde las cuales fundamentar sus prácticas profesionales. Así lo describe un/a entrevistado/a, quien menciona a una profesora, psicóloga sistémica, como la persona clave que la llevó a elegir el área clínica. En otro relato, el eclecticismo teórico fue una opción.

Dentro de los enfoques teóricos presentes en la formación de los/as entrevistados/as, se destaca la influencia de Sergio Yulis y la introducción del conductismo clásico en el laboratorio experimental de la Universidad de Chile:

... llegó un profesor de su doctorado ... que fue el que introdujo el conductismo aquí, era de apellido Yulis y te entusiasmaba porque traía lo último; nosotros que estábamos formados metodológicamente de forma muy estricta se nos facilitó su enseñanza sistemática (participante 4).

Se menciona la figura de otros psicólogos que fueron significativos para los/as entrevistado/as, reconociendo su esfuerzo por diversificar la formación de psicólogos/as de la época y su posterior influencia en la trayectoria profesional:

... un profesor... exiliado ... que estaba en Bélgica, contó un proyecto sobre un trabajo con niños... entonces yo dije, este gallo es feliz en lo que hace porque lo transmitía y él vino a mostrarnos todo lo que era la intervención en redes, un trabajo comunitario ... (participante 1).

... un filósofo con una línea psicoanalítica, que fue el primer profe que yo escuché en mi vida que habló de la posmodernidad ... (participante $5)$.

Luego de egresar de pregrado - en el caso de nuestros participantes entre 1974 y 1994 -, existe una tendencia a seguir perfeccionándose en las distintas áreas de la psicología, ya sea por interés personal en temáticas emergentes o por requerimientos de sus espacios laborales. En el caso de los/as participantes, los estudios fueron diplomados, postítulos y magíster. Uno/a de los/as participantes menciona haber obtenido el grado de magíster en programación neurolingüística, en otros casos una especialización en psicología jurídico-forense y psicoterapia posracionalista, todos obtenidos en universidades y centros de estudios nacionales.

\section{Práctica profesional}

El ejercicio profesional de los participantes se 
desarrolló en diferentes instituciones públicas y privadas. Además, realizaron atención clínica particular, vinculándose de forma inmediata con distintos espacios laborales debido a la necesidad local de contar con psicólogos/as. Las trayectorias laborales de los/as participantes son distintas. En primera instancia, no todos fueron contratados en su área de interés; algunos comenzaron trabajando en la ciudad de su casa de estudio, mientras que otros regresaros a su ciudad de origen:

... una vez hasta hice selección de personal, una sola vez en una empresa [que] me contrató para selección de personal ... no me gustó nada (participante 5).

... mi primera pega fue en un proyecto para la municipalidad de Valparaíso, entonces yo trabajé en un proyecto que se llamaba Casa Blanca (participante 2).

... trabajé diez años en Santiago, principalmente en tres empresas, trabajé allá siempre en el ámbito de la consultoría (participante 3 ).

Hay un factor común en las trayectorias laborales de los participantes: todos se inclinan a la docencia en algún punto de su carrera, algunos complementan su trabajo principal con clases en institutos profesionales y en carreras profesionales distintas a la psicología. Sin embargo, tres de los entrevistados han formado parte importante de la formación de psicólogos/as en la ciudad.

... yo creo que partí en la universidad como en el 2007 o 2008, y no partí con psicología, partí con ingeniería comercial, en gestión de recursos humanos (participante 3 ).

Los entrevistados no muestran interés por el área de investigación, solo uno/a se dedica activamente a investigar con un grupo de trabajo vinculado a una universidad estatal de la capital:

... ahí yo me empecé a meter en el área de investigación, pero ha sido más bien el tema desde donde yo trabajo, que es el tema de salud sexual y reproductiva ... hemos desarrollado otras cosas (participante 2).

Según un/a entrevistado/a, en la ciudad de Los Ángeles la psicología era un ámbito de formación casi inexplorado y la cantidad de psicólogos/as trabajando en ella no era comparable a la cantidad que había en las grandes ciudades del país, además los ciudadanos desconocían esta profesión. Es así como los/as participantes del estudio podrían considerarse como sujetos protagonistas y que grafican el momento histórico de institucionalización de la psicología en la ciudad.

... no había consultoras, no había muchos psicólogos que se dedicaran a lo laboral ... el año 2004 formamos esta empresa con mi socia (participante 3 ).

A pesar del número reducido de psicólogos/as en la ciudad, los participantes reportan que se conformó una agrupación denominada Asociación de Psicólogos Biobío, de la cual, más tarde, se desprendió una segunda agrupación de psicólogos locales quienes dieron paso a la creación de la filial del Colegio de Psicólogos de la región del Biobío, la cual sigue vigente:

... el 2008, en la universidad, se empezó a gestionar el juntar a los psicólogos, no solamente a los que trabajaban en la misma universidad, sino que, de Los Ángeles, para armar un grupo. Y se armó un grupo que, no me acuerdo como se llamaba, ... Psicólogos Biobío, una cosa así, y nos empezamos a juntar y a tener distintos objetivos (participante 5).

Después de un funcionamiento fructífero, los entrevistados exponen la disminución de la participación en la filial del Colegio de Psicólogos. Esto se atribuye a las diferencias políticas con el directorio del colegio, lo cual se expresaría en la poca importancia al trabajo que se realiza a nivel local, la falta de posicionamiento sobre temas de relevancia social y la ausencia de un impacto resolutivo en la toma de decisiones sobre asuntos relevantes para el ejercicio de la profesión en el país.

\section{Discusión y conclusiones}

Las trayectorias biográficas de los psicólogos/as entrevistadas/os, organizadas con base en su formación académica, adscripción teórica y práctica profesional, toman matices muy diversos que se relacionan estrechamente con su historia de vida. Es claro precisar que, de acuerdo con el enfoque biográfico, no se pretende hacer una generalización 
apresurada sobre los relatos de vida de los participantes. Sin embargo, existen aspectos de sus trayectorias personales que articulan un discurso sobre la inserción, institucionalización y profesionalización de la psicología en la ciudad de Los Ángeles. Es decir, los participantes entregan la posibilidad de hacer una aproximación a la historia local de la psicología a partir de su individualidad, en donde su relato toma protagonismo como fuente relevante. En este sentido, se reconoce como limitación de este estudio, la reducida extensión y número de participantes y la incapacidad de generalizar sus resultados. Sin embargo, esto también se puede concebir como una ventaja táctica que permite la reconstrucción de la historia de la psicología con mayor profundidad, contextualizada y que recoja la experiencia cotidiana de participantes anónimos (Man, 2013; Pasek de Pinto, 2006).

En relación con la formación de los participantes, se destaca la influencia de distintos académicos que marcaron su formación profesional, evidenciando correspondencias con la historia de la psicología de las universidades de la capital del país. Tal es el caso de la introducción del conductismo, el cual es relatado por los participantes, a partir de su relación de alumno/a de Sergio Yulis, recordando su orientación experimental y su preferencia por la investigación empírica (Quezada, Vergés, \& Laborda, 2014). También se menciona el desarrollo incipiente de la psicología comunitaria en la Universidad Central, antecedente relevante para el registro histórico de esta subdisciplina. Se destaca la figura de profesores que promovieron una aproximación comunitaria de la psicología novedosa y necesaria, en un delicado momento posdictatorial que requería con urgencia la reconstrucción del tejido social.

La formación de los/as participantes de este estudio se desarrolló en un contexto de dictadura cívico-militar que vulneró y restringió los derechos de las personas a través de la violencia política, por medio de distintas formas de ejercicio del poder. Sin duda, este contexto influyó en la formación de los estudiantes de psicología que, independientemente de su posición política con el régimen, restringía sus posibilidades de reunión, forzó al exilio a sus profesores y persiguió y/o asesinó a compañeros de universidad. El régimen de Pinochet se caracterizó por su impronta neoliberal, lo cual impactó en distintas esferas de la sociedad. En este contexto, comenzó el proceso de privatización de la educación superior en que se fundaron diversas universidades que se separaban de las tradicionales (Villegas, 2016). Asimismo, existía una gran influencia militar en las diferentes casas de estudio (Ligüeño Espinoza \& Parra Moreno, 2007), lo cual se expresaba en que los rectores fueron militares designados; acontecimiento que se extiende hasta el final de la dictadura y que, en ocasiones, se reconoce como matriz de la cultura institucional posterior a esta.

A pesar de las restricciones que imponía la dictadura militar, los estudiantes mantuvieron un rol activo en la organización de instancias académicas. Un ejemplo de esto es el primer encuentro nacional de estudiantes de psicología, el cual se desarrolló en la Universidad de Concepción. Esta actividad es de gran relevancia, no solo porque permitió la profundización en elementos propios de la psicología, sino porque recupera un espacio de intercambio y diálogo en medio de un estado de excepción prohibitivo y violento.

En relación con la adscripción teórica, los participantes refieren la influencia de sus formadores, principalmente en las teorías conductistas y psicoanalíticas. Sin embargo, cada uno siguió un proceso de definición profesional, cuestión que, por medio de la formación de posgrado, fueron legitimando en un área de acción específica bajo influencias teóricas diversas (e.g., posracionalismo, cognitivismo, holístico). Sin embargo, se observa que la adscripción teórica fue cambiando a medida que las distintas instancias laborales lo exigían. Esto deja en evidencia la regulación externa que la psicología contemporánea nacional ha ido incorporando acríticamente, lo cual no solo es asumido como opciones profesionales de adscripción a una teoría explicativa de "lo psicológico", sino que es introducido a las universidades con estatus de verdad por el mercado del trabajo.

Con respecto a la práctica profesional, se puede mencionar que los participantes fueron los primeros psicólogos/as en instalarse en la ciudad en distintos ámbitos de desarrollo profesional: la atención clínica particular, la docencia y la consultoría. Las primeras consultas de atención clínica y laborales fueron instaladas entre la década de 1990 y 2004, mientras que las primeras carreras de psicología se iniciaron el año 2004 en la Universidad Santo Tomás y en la Universidad Bolivariana. Es posible mencionar como una instancia distinta de acción profesional, la fundación y participación de la 
Asociación de Psicólogos del Biobío y posterior filial regional del Colegio de Psicólogos en el Biobío.

Finalmente, los participantes de esta investigación, no siendo personajes relevantes en la historia oficial de la psicología, pueden ser considerados protagonistas anónimos de un proceso de institucionalización de la psicología en un contexto local. Aunque no son personajes excepcionales en la historia de la psicología, se los reconoce en su localidad como actores, en el sentido utilizado por Latour (2008), articulados en una red de relaciones sociotécnicas del dispositivo psicológico.

A partir de lo anterior, se reconoce la historia de la disciplina desde sus participantes, mediante la remembranza de su rol de estudiantes y su trayectoria profesional a partir de sus experiencias; en este caso, muy vinculada a la historia de sus centros de formación universitaria que se ubicaban en ciudades centrales en la administración del saber-poder disciplinar. En este sentido, la reducción de la escala geográfica no asegura que nos estemos refiriendo exclusivamente a los acontecimientos propios de la ciudad, sino que da cuenta de la apropiación, circulación e institucionalización de los saberes psi en acción en un territorio, material y simbólico especifico (Mardones Barrera, 2016b). Esto ha sido recogido, de forma limitada, a partir de la agencia de los entrevistados con relación al despliegue de la disciplina y su contribución a la configuración social del significado de esta a nivel local, no entendida como la reificación de una verdad, sino que como configuraciones provisorias y en constante revisión.

Estos elementos revelan la importancia de una comprensión de la historia de la psicología como historia local que no desconoce su vertiente sociocultural, con la ventaja de reportar puntos, hasta ahora, anónimos de una red de relaciones que configuran la psicología nacional. Sin duda, el considerar solo el relato de los participantes es una limitación para describir esta red de relaciones, cuestión que se presenta como desafío para trabajos posteriores.

\section{Referencias}

Aguilar, G. (1983). Historia de la psicología en Bolivia. Revista Latinoamericana de Psicología, 15(3), 311325.

Aguirre Rojas, C. A. (2003). El queso y los gusanos: un modelo de historia crítica para el análisis de las culturas subalternas. Revista Brasileira de História, 23(45), 71-101.

Recuperado de https://bit.ly/2AqLVoy

Alarcón, R. (1980a). Desarrollo y estado actual de la psicología en el Perú. Revista Latinoamericana de Psicología, 12(2), 205-235.

Recuperado de https://bit.ly/2ApNmU4

Alarcón, R. (1980b). Walter Blumenfeld (1882-1967). Revista Latinoamericana de Psicología, 12(2), 378379

Recuperado de https://bit.ly/2CKrmoD

Ardila, R. (1973). La psicología en Colombia: desarrollo histórico. Ciudad de México, México: Trillas.

Ardila, R. (1988). Mercedes Rodrigo (1891-1982). Revista Latinoamericana de Psicología, 20(3), 429-434 Recuperado de https://bit.ly/2TnQeIg

Arias Gallegos, W. L. (2016). Contribuciones para una historia local de la psicología en Arequipa. En R. E. Mardones Barrera (Ed.), Historia local de la psicología: discusiones teóricas, metodológicas y experiencias de investigación (pp. 29-47). Santiago, Chile: Ril editores.

Barboza-Palomino, M. (2016). La formación del psicólogo en la Universidad Nacional Mayor de San Marcos: contraste de dos planes de estudios. Revista Peruana de Historia de la Psicología, 2, 73-87. Recuperado de https://bit.ly/2R7BZdK

Barrero C., E. (2012). Del discurso encantador a la praxis liberadora. Psicología de la liberación: aportes para la construcción de una psicología desde el Sur. Bogotá, Colombia: Ediciones Cátedra Libre.

Benites Morales, L. \& Zapata Ponce, L. (2009). La psicología en el Perú: formación académica y ejercicio profesional. Psicología para América Latina, 17. Recuperado de https://bit.ly/2F5OaAz

Bravo, L. (2004). Cincuenta años de psicología en la Universidad Católica. Psykhe, 13(1), 197-204. http://dx.doi.org/10.4067/S071822282004000100016

Bravo-Valdivieso, L. y Tschorne Tetelman, P. (1969). La psicología en Chile. Revista Latinoamericana de Psicología, 1(2-3), 95-104.

Recuperado de https://bit.ly/2AuQ1fb

Campos, R. H. F. (2003). Helena Antipoff: Razão e sensibilidade na psicologia e na educação. Estudos Avançados, 17(49), 209-231.

https://dx.doi.org/10.1590/S010340142003000300013

Dagfal, A. (2014). Breve historia de la Psicología en la ciudad de La Plata (1906-1966). Universitas Psychologica, 13(5), 1759-1775.

http://dx.doi.org/10.11144/Javeriana.upsy13-5.bhpc

Danziger, K. (1979). The social origins of modern psychology. En A. R. Buss (Ed.), Psychology in social context (pp. 25-44). New York: Irvington.

De la Torre Molina, C. L. (2009). Historia de la 
psicología en Cuba: cincuenta años de psicologíacincuenta años de revolución. Psicología para América Latina, 17.

Recuperado de https://bit.ly/2RyuITW

Gallegos, M. (2005). Cincuenta años de historia de la psicología como institución universitaria en Argentina. Revista Latinoamericana de Psicología, 37(3), 641-652.

Recuperado de https://bit.ly/2AuQvlv

Gallegos, M. (2012). Historia de la psicología interamericana: Sociedad Interamericana de Psicología (1951). Revista Psychologia Latina. 3(1), 23-36.

Recuperado de https://bit.ly/2TqT9Qq

García, J. E. (2007). La psicología en Paraguay y el problema de la determinación de los pioneros. Revista Intercontinental de la Psicología y Educación. 9(2), 113-146.

Recuperado de https://bit.ly/2Qkqk63

García, J. E. (2010). La cátedra de Psicología Experimental en la Universidad Católica de Asunción: 1985-1987. Revista Interamericana de Psicología, 44(1), 157-167.

Recuperado de https://bit.ly/2TjAu8Q

García, J. E. (2011). La enseñanza de la historia de la psicología en las universidades paraguayas. Revista de Investigación en Psicología, 14(2), 71-94.

Recuperado de https://bit.ly/2RvTmnW

García, J. E. (2012). Historia y estado actual de la psicología en la ciudad de Encarnación, Paraguay. Fundamentos en Humanidades, 12(24), 37-88.

Recuperado de https://bit.ly/2ArGl5f

Ginzburg, C. (1999). El queso y los gusanos. El cosmos, según un molinero del siglo XVI. Barcelona, España: Muchnik Editores.

Hoffmann, M. H., Tortosa, F., \& Carbonell Vayá, E. (1994). Emilio Mira y López y el desarrollo de la psicología del tránsito. Los casos de España y Brasil. Revista Latinoamericana de Psicología, 26(3), 495516.

Recuperado de https://bit.ly/2Su7ETn

Klappenbach, H. (1994). La recepción de Wundt en la Argentina. 1907: creación del segundo curso de psicología en la Universidad de Buenos Aires. Revista de Historia de la Psicología. 15(1-2), 181-197.

Klappenbach, H. (2011). Historias locales de la psicología. Plácido Horas y las primeras investigaciones en psicología en San Luis. Argentina. Memorandum. Mémoria e História em Psicologia, 21, 62-74.

Recuperado de https://bit.ly/2Arracg

Klappenbach, H. (2016). Enfoques cuantitativos y cualitativos en la investigación histórica de las psicologías nativas y locales. En R. E. Mardones Barrera (Ed.), Historia local de la psicología: discusiones teóricas, metodológicas y experiencias de investigación (pp. 97-122). Santiago, Chile: RIL Editores.
Laborda, M. A. \& Quezada, V. E. (Eds.) (2010). Notas históricas de la psicología en Chile. Santiago, Chile: Editorial Universitaria.

Latour, B. (2008). Reensamblar lo social. Una introducción a la teoría del actor-red. Buenos Aires, Argentina: Manantial.

León, R. (1982). Los psicoanalistas latinoamericanos y la difusión de sus trabajos en la revista "Internationale Zeitschrift für psychoanalyse". Un estudio bibliométrico. Revista Latinoamericana de Psicología. 14(2), 171-182.

Recuperado de https://bit.ly/2AqHIRC

Ligüeño Espinoza, S. \& Parra Moreno, D. (2007). La psicología en la universidad de Chile: una propuesta de estudio para comprender la historia de la psicología en Chile. Cuadernos de Neuropsicología, 1(3), 223-235.

Recuperado de https://bit.ly/2F51s0T

Luco, A. (2010). La psicología en Chile: ciencia, disciplina y profesión. Santiago, Chile: Catalonia.

Luco, A. (2016). El rol del Colegio de Psicólogos durante la dictadura cívico-militar. Revista de Psicología, 25(1), 1-8. http://dx.doi.org/10.5354/0719-0581.2016.42246

Mallimaci, F. \& Giménez Béliveau, V. (2006). Historia de vida y métodos biográficos. En I. Vasilachis de Gialdino (Coord.), Estrategias de investigación cualitativa (175-212). Barcelona, España: Editorial Gedisa.

Maluf, M. (2012). Sociedad Interamericana de Psicología: historia, trayectoria y proyectos. Revista de psicología. 30(1), 215-220.

Recuperado de https://bit.ly/2s7z5Xq

Man, R. (2013). La microhistoria como referente teórico-metodológico. Un recorrido por sus vertientes y debates conceptuales. Historial Actual Online, 30, 167-173.

Recuperado de https://bit.ly/2R9ilzt

Mardones, R. E., Ulloa Martínez, J. B., \& Salas, G. (2018). Usos del diseño metodológico cualitativo en artículos de acceso abierto de alto impacto en ciencias sociales. Forum Qualitative Sozialforschung/Forum: Qualitative Social Research, 19(1), 1-18.

http://dx.doi.org/10.17169/fqs-19.1.2656Mardones Barrera, R. E. (2016a). Historia local de la psicología. Discusiones teóricas, metodológicas y experiencias de investigación. Santiago, Chile: Ril editores.

Mardones Barrera, R. E. (2016b). Discusiones epistémicas sobre la dimensión local en las ciencias sociales. Perspectivas para la historización de la psicología en América Latina. En R. E. Mardones Barrera (Ed.), Historia local de la psicología: discusiones teóricas, metodológicas y experiencias de investigación (pp. 29-47). Santiago, Chile: Ril editores.

Pasek de Pinto, E. (2006). ¿Cómo construir categorías 
en microhistoria? Revista de Artes y Humanidades UNICA, 7(16), 85-97.

Recuperado de https://bit.ly/2H8V5tH

Pérez-Luco Arenas, R., Lagos Gutiérrez, L., Mardones Barrera, R., \& Sáez Ardura, F. (2017). Taxonomía de diseños y muestreo en investigación cualitativa. Un intento de síntesis entre las aproximaciones teórica y emergente. Ámbitos. Revista Internacional de Comunicación, 39.

Recuperado de https://bit.ly/2AuXGdl

Poblete, M. (1980). A propósito del centenario de la creación del primer laboratorio de psicología experimental. Revista Chilena de Psicología, 3(1), 15-19.

Polanco, F. \& Lopes Miranda, R. (2014). Recepción del conductismo en Argentina y Brasil: un estudio de dos universidades, 1960-1970. Universitas Psychologica, 13(5), 2035-2045.

http://dx.doi.org/10.11144/Javeriana.upsy13-5.rcab

Pujadas, J. J. (2000). El método biográfico y los génerso de la memoria. Revista de Antopología Social, 9, 127-158.

Recuperado de https://bit.ly/2EmaJiq

Quezada, V. E., Vergés, Á. \& Laborda, M. A. (2014). Sergio Yulis: pasado y presente del enfoque conductual en Chile. Psykhe, 23(1), 1-11.

http://dx.doi.org/10.7764/psykhe.23.1.527

Rodrigues Soares, A. (2010). A Psicologia no Brasil. Psicologia: Ciência e Profissão, 30, 8-41.

Recuperado de https://bit.ly/2QpaN5e

Rodríguez Arias, E. (2009). Breve historia de la psicología en República Dominicana. Psicología para América Latina, 17, Recuperado de https://bit.ly/2EDoNpe

Rossi, L. A., Ibarra, F., \& Ferro, C. (2009). Historia de la psicología en la Argentina. Psicología para América Latina, 17.

Recuperado de https://bit.ly/2Xvg5kn

Ruperthuz Honorato, M. (2014). Germán Greve Schlegel y la recepción del psicoanálisis en Chile: la historia de un médico chileno "probablemente alemán”. Universitas Psychologica, 13(5), 1847-1867. http://dx.doi.org/10.11144/Javeriana.upsy13-5.ggsr

Ruperthuz Honorato, M. (2015). Freud y los chilenos. Santiago, Chile: Pólvora editorial

Saforcada, E. (2008). La psicología en Argentina: desarrollo disciplinar y realidad nacional. Revista Interamericana de Psicología, 42(3), 462-471.

Recuperado de https://bit.ly/2VspKan

Salas Contreras, G. (2012). La influencia europea en los inicios de la historia de la psicología en Chile. Revista Interamericana de Psicología, 46(1), 99-110. Recuperado de https://bit.ly/2BYLqSw

Salas, G. (2013). Juan Serapio Lois (1844-1913): pionero de la psicología científica en Chile. Psykhe, 22(1), 111-123.

http://dx.doi.org/10.7764/psykhe.22.1.630
Salas, G. (2016). Historiografía, epistemología y enunciados sobre la historia de la psicología con consideraciones sobre lo local. En R. E. Mardones Barrera (Ed.), Historia local de la psicología: discusiones teóricas, metodológicas y experiencias de investigación (pp. 97-122). Santiago, Chile: Ril editores.

Salas, G., Mardones, R., Gallegos, M., \& Ponce, F. P. (2014). Amanda Labarca y sus referencias psicológicas en el contexto educativo en Chile. Universitas Psychologica, 13(5), 2059-2068. http://dx.doi.org/10.11144/Javeriana.upsy13-5.alrp

Salas, G. \& Lizama, E., (2013). Historia de la psicología en Chile 1889-1981 ( $2^{\mathrm{a}}$ ed.). La Serena, Chile: Universidad de La Serena.

Salinas Villacrés, D. E. \& Prado Cabrera, K. D. (2017). La formación del psicólogo clínico en Cuenca-Ecuador (Tesis de licenciatura). Universidad de Cuenca, Cuenca, Ecuador.

Sanmartín Ascaso, J. (1995). Macrohistoria, microhistoria o historia. Scripta Fulgentina. 9(10), 29-36.

Recuperado de https://bit.ly/2GSwjj9

Sanz Hernández, A. (2005). El método biográfico en investigación social: potencialidades y limitaciones de las fuentes orales y los documentos personales. Universidad de Zaragoza. 57(1), 99-115.

Recuperado de https://bit.ly/2ReMk7b

Scherman, P. (2014). Inicios de la psicología en Córdoba: la enseñanza en el Instituto de Filosofía. Universitas Psychologica, 13(5), 2069-2077.

http://dx.doi.org/10.11144/Javeriana.upsy13-5.ipce

Schulmeyer, M. K. (2013). 20 años de psicología en las universidades privadas de Bolivia. Ajayu, 11(2), 118-137.

Recuperado de https://bit.ly/2F7J5YE

Taylor, S. J. \& Bogdan, R. (1994). Introducción a los métodos cualitativos de investigación. La búsqueda de significados. Barcelona, España: Paidos.

Toro, J. P. \& Villegas, J. F. (Eds.) (2001). Problemas centrales para la formación académica y el entrenamiento profesional del psicólogo en las Américas (Vol. I). Buenos Aires, Argentina: SIP.

Valles, M. S. (2007). Técnicas cualitativas de investigación social. Reflexión metodológica y práctica profesional. Madrid, España: Síntesis.

Ventura, M. (2004). Psicoanálisis y universidad: un estudio en la carrera de psicología de la Universidad Nacional de Tucumán (UNT). XI Jornadas de Investigación, Universidad de Buenos Aires, Buenos Aires, Argentina.

Vetö Honorato, S. (2013). Psicoanálisis en estado de sitio. La desaparición de Gabriel Castillo y las politicas de psicoanálisis en Chile durante la dictadura militar. Santiago, Chile: Facso/El Buen Aire.

Vía Orellana, F. (2000). Historia y formación del psicólogo en Bolivia. Revista Ciencia y Cultura, 8, 51-62. 
Recuperado de https://bit.ly/2sa4856

Villegas, J. F. (2016). Epílogo. En R. E. Mardones Barrera (Ed.), Historia local de la psicología: discusiones teóricas, metodológicas y experiencias de investigación (pp. 389-400). Santiago, Chile: Ril editores.

Villegas, J. F., Marassi, P., \& Toro, J. P. (Eds.). (2003a). Problemas centrales para la formación académica y el entrenamiento profesional del psicólogo en las Américas (Vol. II). Santiago, Chile: SIP.

Villegas, J. F., Marassi, P., \& Toro, J. P. (Eds.). (2003b). Problemas centrales para la formación académica y el entrenamiento profesional del psicólogo en las Américas (Vol. III). Santiago, Chile: SIP.

Winkler, M. I. \& Reyes-Espejo, M. I. (2015). Historias de mujeres en la psicología chilena: contribuciones de Lola Hoffmann, Héliettè Saint Jean y Vera Kardonsky. Psykhe, 24(1), 1-11. http://dx.doi.org/10.7764/psykhe.24.1.658

Winkler, M. I. (2007). Pioneras sin monumentos: mujeres en psicología. Santiago, Chile: LOM-Universidad de Santiago de Chile.

Fecha de recepción: 24 de noviembre de 2016 Fecha de aceptación: 14 de septiembre de 2018 